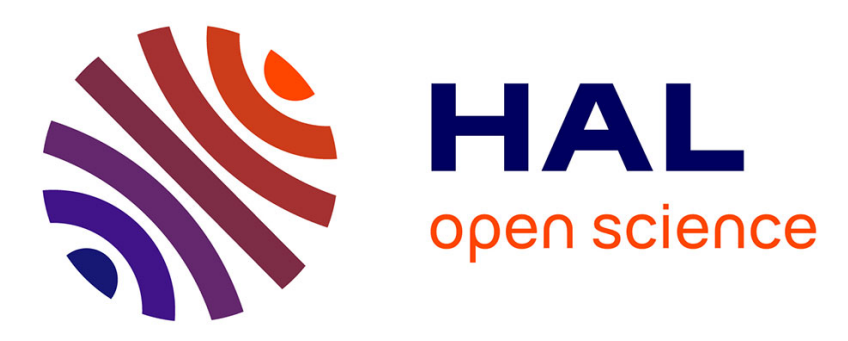

\title{
The Sunward Electron Deficit: A Telltale Sign of the Sun's Electric Potential
}

\author{
J. Halekas, Laura Berčič, P. Whittlesey, D. Larson, R. Livi, Matthieu \\ Berthomier, J. Kasper, A. Case, M. Stevens, S. Bale, et al.
}

\section{- To cite this version:}

J. Halekas, Laura Berčič, P. Whittlesey, D. Larson, R. Livi, et al.. The Sunward Electron Deficit:

A Telltale Sign of the Sun's Electric Potential. The Astrophysical Journal, 2021, 916 (1), pp.16. 10.3847/1538-4357/ac096e . hal-03324152

\section{HAL Id: hal-03324152 \\ https://hal.science/hal-03324152}

Submitted on 18 Nov 2021

HAL is a multi-disciplinary open access archive for the deposit and dissemination of scientific research documents, whether they are published or not. The documents may come from teaching and research institutions in France or abroad, or from public or private research centers.
L'archive ouverte pluridisciplinaire HAL, est destinée au dépôt et à la diffusion de documents scientifiques de niveau recherche, publiés ou non, émanant des établissements d'enseignement et de recherche français ou étrangers, des laboratoires publics ou privés. 


\title{
The Sunward Electron Deficit: A Telltale Sign of the Sun's Electric Potential
}

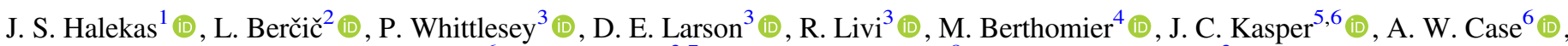 \\ M. L. Stevens ${ }^{6}$ (D), S. D. Bale ${ }^{3,7}$ (D) R. J. MacDowall ${ }^{8}$ (D) , and M. P. Pulupa ${ }^{3}$ (D) \\ ${ }^{1}$ Department of Physics and Astronomy, University of Iowa, Iowa City, IA 52242, USA; jasper-halekas@uiowa.edu \\ ${ }^{2}$ Mullard Space Science Laboratory, University College London, Dorking, RH5 6NT, UK \\ ${ }^{3}$ Space Sciences Laboratory, University of California, Berkeley, CA 94720, USA \\ ${ }^{4}$ Laboratoire de Physique des Plasmas, CNRS, Sorbonne Universite, Ecole Polytechnique, Observatoire de Paris, Universite Paris-Saclay, Paris, F-75005, France \\ ${ }^{5}$ Climate and Space Sciences and Engineering, University of Michigan, Ann Arbor, MI 48109, USA \\ ${ }^{6}$ Smithsonian Astrophysical Observatory, Cambridge, MA 02138, USA \\ ${ }^{7}$ Physics Department, University of California, Berkeley, CA 94720, USA \\ ${ }^{8}$ NASA/Goddard Space Flight Center, Greenbelt, MD 20771, USA \\ Received 2021 May 27; revised 2021 June 7; accepted 2021 June 7; published 2021 July 21
}

\begin{abstract}
As the Parker Solar Probe explores new regions of the inner heliosphere, it travels ever deeper into the electric potential of the Sun. In the near-Sun environment, a new feature of the electron distribution emerges, in the form of a deficit in the sunward suprathermal population. The lower boundary of this deficit forms a cutoff in phase space, at an energy determined by the electric potential drop between the observation point and the outer heliosphere. We explore the characteristics of the sunward deficit and the associated cutoff, as well as the properties of the plasma in which we observe them. The deficit occurs in $\sim 60 \%-80 \%$ of electron observations within $\sim 0.2$ au, and even more frequently in plasma with low $\beta$, low collisional age, and a more anisotropic electron core population. At greater distances, the deficit rapidly disappears, as the suprathermal halo grows, with these two trends likely related. The cutoff energy varies linearly with the local electron core temperature, confirming a direct relationship to the ambipolar electric potential. Meanwhile, the cutoff width varies with $\beta$ and collisional age, suggesting that energy diffusion plays a role in erasing the deficit. The nearly ubiquitous occurrence of the sunward deficit in the inner heliosphere suggests that we may need to reconsider the functional forms commonly used to represent electron distributions in this environment.
\end{abstract}

Unified Astronomy Thesaurus concepts: Solar wind (1534); The Sun (1693)

\section{Introduction}

Since the prediction and subsequent discovery of the solar wind over 60 years ago (Parker 1958; Gringauz et al. 1960; Neugebauer \& Snyder 1962), the scientific community has considered a wide range of theories for its acceleration. Many of these, including the original hydrodynamic Parker model (Parker 1965) and a broad class of "exospheric" models (Jockers 1970; Lemaire \& Scherer 1971, 1973; Pierrard \& Lemaire 1996; Maksimovic et al. 1997; Zouganelis et al. 2004), directly involve the electron pressure gradient and/or the associated electric field. Even for solar wind models that primarily rely on other acceleration mechanisms (e.g., waves), a significant interplanetary electric field still naturally arises as a consequence of the Sun's gravitational field and the presence of a hot plasma in the corona. Quite generally, given that $m_{i} \gg m_{e}$, an electric field must exist to maintain quasi-neutrality in the presence of a gravitational field (Pannekoek 1922). Moreover, global quasi-neutrality requires zero current along a flux tube, which implies an even stronger electric field (Lemaire \& Scherer 1971), given $T_{e} \simeq T_{i}$ and thus $v_{t h_{e}} \gg v_{t h_{i}}$. From a more collective point of view, the electron pressure gradient between the hot corona and the cooler and less dense solar wind implies the presence of a significant ambipolar electric field. As Parker himself has shown (Parker 2010), in the limit of $m_{e} \rightarrow 0$, the exospheric model becomes equivalent to the hydrodynamic model. Given all these considerations, the existence of a significant electric potential drop between the corona and the outer heliosphere seems in little doubt. However, with most previous observations made outside of the region with the majority of the electric potential drop, we have previously only inferred its presence.
Complicating matters, the electron velocity distributions (VDFs) observed at distances of $\gtrsim 0.3$ au appear heavily processed. Solar wind VDFs do not have a purely isotropic Maxwellian form, instead generally consisting of a nearly Maxwellian core, a hotter and more tenuous halo, and a field-aligned strahl (Feldman et al. 1975; Rosenbauer et al. 1977; Pilipp et al. 1987; Maksimovic et al. 2005; Štverák et al. 2009). However, we generally observe much smaller departures from isotropy than one would predict from adiabatic motion in the diverging solar magnetic field, indicating significant alteration of the VDFs by plasma instabilities and/or Coulomb collisions. Several lines of evidence, including an increase in the relative fractional density of the halo with heliocentric distance (Maksimovic et al. 2005; Štverák et al. 2009; Halekas et al. 2020), as well as an increase in the width of the strahl (Hammond et al. 1996; Graham et al. 2017; Berčič et al. 2019), support a scenario wherein the initially free-streaming strahl evolves to form the halo (Landi et al. 2012; Berčič et al. 2020, 2021). On the other hand, the halo may result at least in part from a sunward runaway process driven by the interplanetary electric field (Scudder 2019).

Numerous processes may alter the electron VDFs between the corona and interplanetary space, including Coulomb collisions (Scudder \& Olbert 1979a, 1979b; Salem et al. 2003; Landi et al. 2012; Boldyrev \& Horaites 2019; Horaites et al. 2019; Berčič et al. 2021) and a variety of plasma instabilities, such as electrostatic modes (Gary 1978; Roberg-Clark et al. 2018; López et al. 2020), quasi-parallel whistler modes (Gary et al. 1975, 1994, 1999; Saeed et al. 2017; Shaaban et al. 2018; López et al. 2019), oblique whistler/magnetosonic instabilities (Horaites et al. 2018; Vasko et al. 2019; Verscharen et al. 2019; Micera et al. 2020), and the 


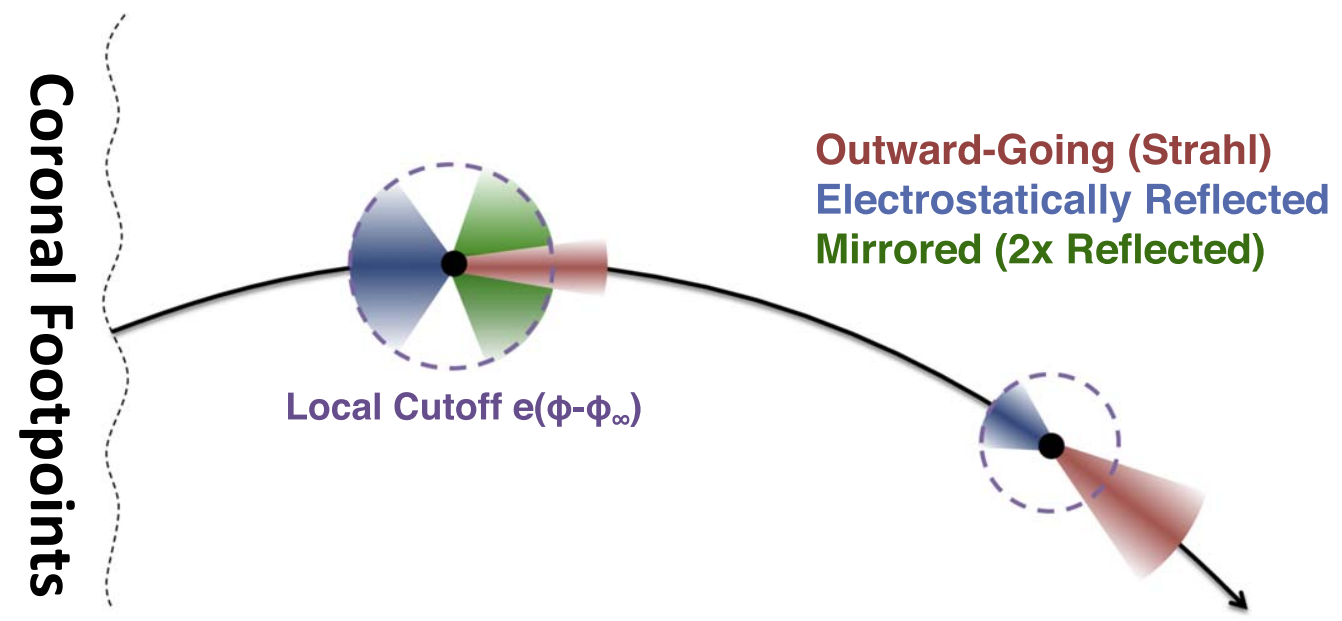

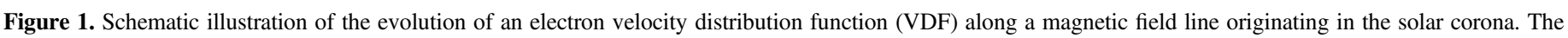

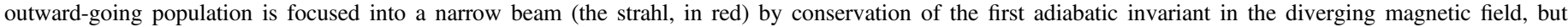

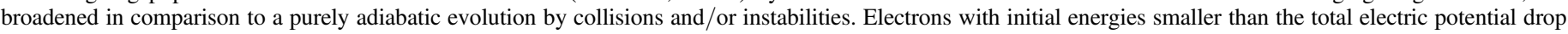

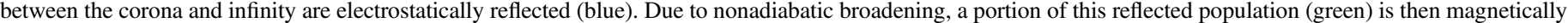
mirrored and trapped.

firehose instability (Shaaban et al. 2018; Innocenti et al. 2020). New observations from the Parker Solar Probe (PSP) (Fox et al. 2016) have provided recent support for the importance of whistler waves, particularly oblique modes (Cattell et al. 2021; Jagarlamudi et al. 2021; Halekas et al. 2021). However, it appears highly likely that multiple mechanisms may play a role, possibly through a multi-step process (Micera et al. 2020). In any case, the operation of processes that violate magnetic moment conservation, together with the presence of the interplanetary electric field, naturally leads to the formation of a trapped population of electrons (see Figure 1), excluded from larger heliocentric distances by the electric field and from smaller distances by the magnetic mirror force (Horaites et al. 2019; Boldyrev et al. 2020). This trapping mechanism likely plays a significant role in the formation of the core, and possibly also the halo.

The electrostatic reflection of the low-energy electrons by the interplanetary electric field should produce observable signatures in the VDF, in particular a cutoff in the sunward portion of the VDF at an energy equal to the integrated electric potential drop between the observation point and infinity (see Figure 1). At heliocentric distances of $\gtrsim 0.3 \mathrm{au}$, signatures of the sunward deficit do not obviously persist, though some suggestive observations exist (Pilipp et al. 1987). Presumably collisions and/or instabilities erase the signature of the sunward deficit as they isotropize the distribution and modify the strahl and halo. At the same time, the cutoff moves to lower energies at greater heliocentric distances, possibly further obscuring its presence.

Recently, near the perihelion of PSP, the Solar Probe ANalyzer-Electrons (SPAN-E) sensors of the Solar Wind Electrons Alphas and Protons (SWEAP) instrument suite (Kasper et al. 2016; Whittlesey et al. 2020) have for the first time identified a clear deficit (with respect to a drifting bi-Maxwellian) in the sunward suprathermal electron population (Berčič et al. 2020; Halekas et al. 2020, 2021), with a form consistent with that predicted by simulations (Berčič et al. 2021). In this manuscript, we explore the characteristics of the sunward electron deficit, and the properties of the intervals during which we can observe clear signatures of the deficit.

\section{The Sunward Deficit}

We identify the sunward electron deficit by comparing the VDFs measured by SPAN-E (Whittlesey et al. 2020) to drifting bi-Maxwellian functional fits to the core population. We follow the procedure developed in Halekas et al. (2020), which simultaneously fits the core and the secondary electron contamination generally present at low energy. We rely on a cross-calibration to quasithermal noise measurements from FIELDS (Moncuquet et al. 2020) to determine the absolute sensitivity of the SPAN-E sensors (assumed constant), and perform a daily relative sensitivity calibration across all look directions by enforcing electron gyrotropy in the plasma frame. We utilize proton velocity measurements from either the Solar Probe Analyzer-Ions (SPAN-I; Kasper et al. 2016) or the Solar Probe Cup (Case et al. 2020), depending on which instrument better observes the proton core, to shift the electron VDF into the proton frame. We also utilize magnetometer data from the FIELDS suite (Bale et al. 2016) to organize the VDF by pitch angle. We then fit to the portion of the VDF for $v \lesssim 2 v_{\text {th }}$, excluding velocities within $45^{\circ}$ of the strahl direction (as determined from the suprathermal pitch angle distribution).

Figure 2 shows a representative VDF and the corresponding core fit. The core fit broadly matches the observations at low velocities. At moderate and high velocities the anti-sunward (anti-field aligned in this case) portion of the VDF extends above the core fit, due to the presence of the strahl. At moderate velocities, the sunward portion of the VDF drops below the core fit, before rising above it again at high velocities. The rise at high velocities results from the presence of a weak halo population, as well as instrumental background counts from penetrating cosmic rays and/or natural radioactivity in the microchannel plate detectors.

The ratio between the observed VDF and the core fit more clearly reveals small differences between the two. At low velocities, a residual signature of secondary electron contamination remains; this low-energy signal is ubiquitous in the inner heliosphere where the primary electron energy is high enough to generate significant secondary emission from instrument and spacecraft surfaces (Whittlesey et al. 2020; Halekas et al. 2020), and it has no physical significance. At velocities just below the 

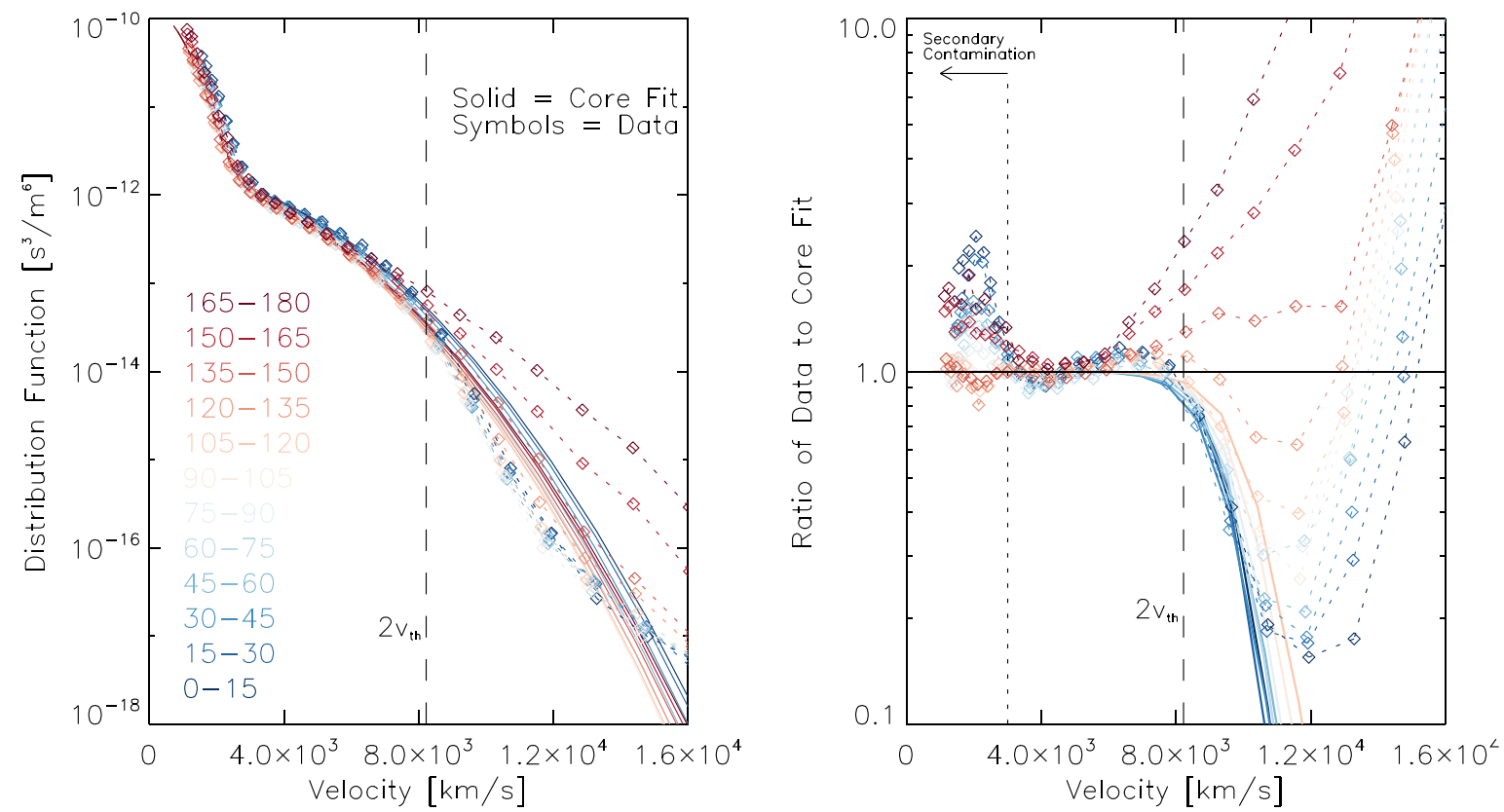

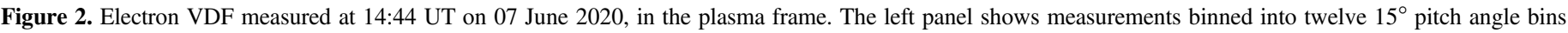

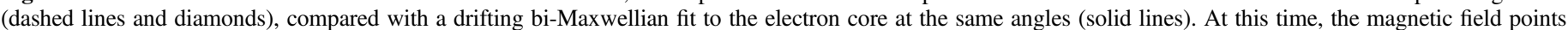

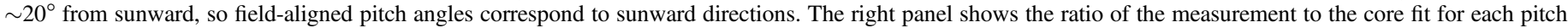

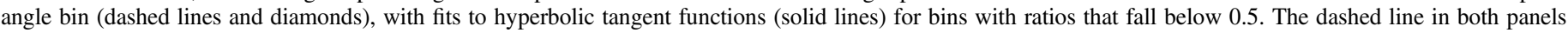
marks twice the thermal velocity.

observed deficit, the VDF slightly exceeds the core fit in both the sunward and anti-sunward directions. This excess results from the presence of the strahl in the anti-sunward direction, and most likely from the electrostatically reflected portion of the strahl in the sunward direction. The deficit in the VDF clearly appears across a broad range of angles, extending even into the slightly antisunward angles. The $\gtrsim 90^{\circ}$ range of the deficit presumably results from a combination of scattering and magnetic mirroring, as shown in Figure 1.

For each pitch angle bin where the ratio between the observed VDF and the core fit falls below 0.5, we fit the portion of the VDF at velocities above the secondary contamination and below the velocity with the minimum ratio to a hyperbolic tangent function of the form $f_{\text {cut }}(v)=0.5 \times$ $\left(1+\tanh \left[\left(v_{c}-v\right) / w\right]\right)$ to estimate the local cutoff velocity $v_{c}$ and cutoff width $w$. The hyperbolic tangent function provides a convenient empirical functional form to characterize the location and width of the cutoff, and it has been used for similar purposes at shocks (Bale et al. 2005). A complementary approach to determining $v_{c}$, which yields comparable results in the sunward direction, has been developed by Berčič et al. (2021a). Figure 2 shows examples of our functional fits, for each pitch angle bin with an observable deficit. We find similar cutoff velocities and widths for all pitch angle bins with an observable deficit, but with a slight trend toward higher cutoff velocities for pitch angles farther from sunward. This appears somewhat contrary to what one might expect based on the simple schematic of Figure 1, and may indicate the presence of a significant locally mirroring population. Though we have no obvious explanation for the origin of this trend, it shows up clearly not just in the fits but also in the observations.

We repeat the analysis described above for every electron VDF measured by SPAN-E for which we obtain a good core fit ( $\sim 86 \%$ of all measured VDFs), for the first seven PSP orbits.
We show a sample of the results from the fifth PSP perihelion, together with contextual information, in Figure 3. We find that the magnitude of the cutoff velocity increases with decreasing heliocentric distance, consistent with an origin related to the interplanetary electric field. The cutoff velocity reaches $\sim 10,000 \mathrm{~km} \mathrm{~s}^{-1}$ near perihelion, corresponding to a potential drop of $\sim 285 \mathrm{~V}$ between the observation location and the outer heliosphere. When present, the deficit often extends from sunward through locally mirroring angles and slightly into the anti-sunward angles, as in the example from Figure 2, and consistent with the schematic illustration of Figure 1. During a few intervals, the deficit appears more confined to the most nearly sunward angles. As expected, the deficit never appears in the anti-sunward direction, which contains the strahl. The magnitude of the cutoff velocity at any given time remains nearly constant as a function of pitch angle, though we sometimes see the same slightly increasing trend with angle from the sunward direction noted for the example in Figure 2.

Though we commonly find signatures of the sunward deficit near the perihelion of PSP, we do not observe them (at least not at the 0.5 threshold level that we have chosen) at every instant. Figure 3 shows that the occurrence of the deficit appears to correlate with other properties of the electron VDF. In particularly, we most commonly observe the deficit at times with less scattering apparent in the suprathermal electron pitch angle distributions, a more anisotropic core distribution, a lower fractional halo density, a smaller electron $\beta_{\|}$, and a lower collisional age (which we estimate in the same manner as Salem et al. (2003), but assuming $r_{0}=0.05$ au and a temperature exponent $\alpha=0.5$ ). These trends all suggest that the processes that act to isotropize the electron VDF and produce the halo also remove the deficit signature, or at least that they operate on similar timescales to the processes that erase the deficit. The formation of the halo in particular should act to "fill in" and thus obscure the deficit signature, so the anticorrelation between time periods with 

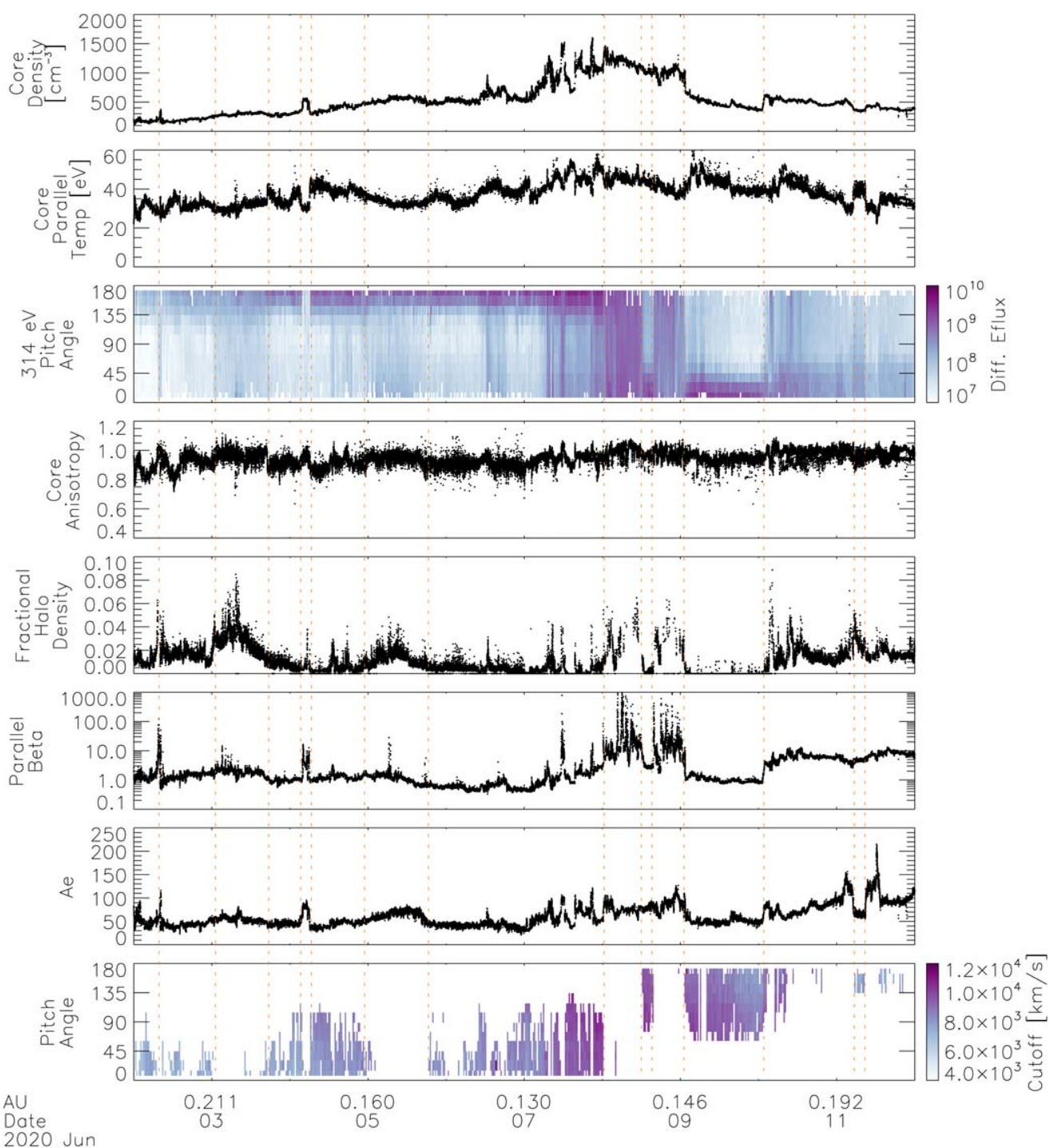

Figure 3. Observations from the fifth Parker Solar Probe perihelion. The eight panels show electron core density and parallel temperature from bi-Maxwellian fits, electron pitch angle distribution at $314 \mathrm{eV}$ (in the plasma frame), core anisotropy $\left(T_{\perp} / T_{\|}\right)$from bi-Maxwellian fits, fractional halo density, electron $\beta_{\|}$, an estimate of the collisional age $A e$, and the local sunward electron cutoff velocity as a function of pitch angle. Orange lines indicate approximate boundaries between flux tubes containing plasma with different characteristics.

significant halo densities and the presence of the deficit appears physically reasonable. We will further explore these trends in Section 3.

To validate the tentative identification of the sunward deficit as a signature of the interplanetary electric field, we explore the joint distributions of the sunward cutoff velocity with other basic solar wind properties in Figure 4. We find that the cutoff has characteristics entirely consistent with those expected for that associated with the Sun's electric potential. The average observed cutoff velocity, which ranges from 4,000-10,000 km $\mathrm{s}^{-1}$, decreases monotonically with distance, increases monotonically with electron core density and temperature, and decreases with local solar wind bulk speed.

The observed dependence of the cutoff velocity on electron core temperature provides the clearest confirmation of a correspondence with the interplanetary electric field. One can utilize the generalized Ohm's law to predict a linear relationship between the interplanetary electric potential and the local electron core temperature, assuming that the electron pressure gradient term $-\nabla P_{e} /\left(e n_{e}\right)$ dominates the parallel electric field.
Scudder \& Olbert (1979b) suggested a slope of $\sim 7$ between the local values of the electric potential and the electron core temperature, consistent with the electron density and core temperature scaling as $n_{e} \propto r^{-2}$ and $T_{c} \propto r^{-1 / 3}$. Our data clearly support a linear relationship, but with a slope of closer to $\sim 5$, corresponding to a range of potentials of $50-300 \mathrm{~V}$ for electron core temperatures of $\sim 10-60 \mathrm{eV}$. This slope implies $T_{c} \propto r^{-0.5}$, or a slightly larger temperature exponent if the density falls off faster than $r^{-2}$ (i.e., if the solar wind is still accelerating), entirely consistent with observations in the inner heliosphere from Helios and PSP (Maksimovic et al. 2005; Halekas et al. 2020). This result also roughly agrees with the recent prediction $T_{c} \propto r^{-2 / 5}$ from Boldyrev et al. (2020).

The anticorrelation between the cutoff velocity and the solar wind bulk speed shown by Figure 4 may appear surprising, but it naturally arises from the anticorrelation between electron temperature and solar wind speed present in the inner heliosphere, which presumably results from the initial conditions in the corona (Maksimovic et al. 2020; Halekas et al. 2020). This observed trend does indicate that the locally slower 

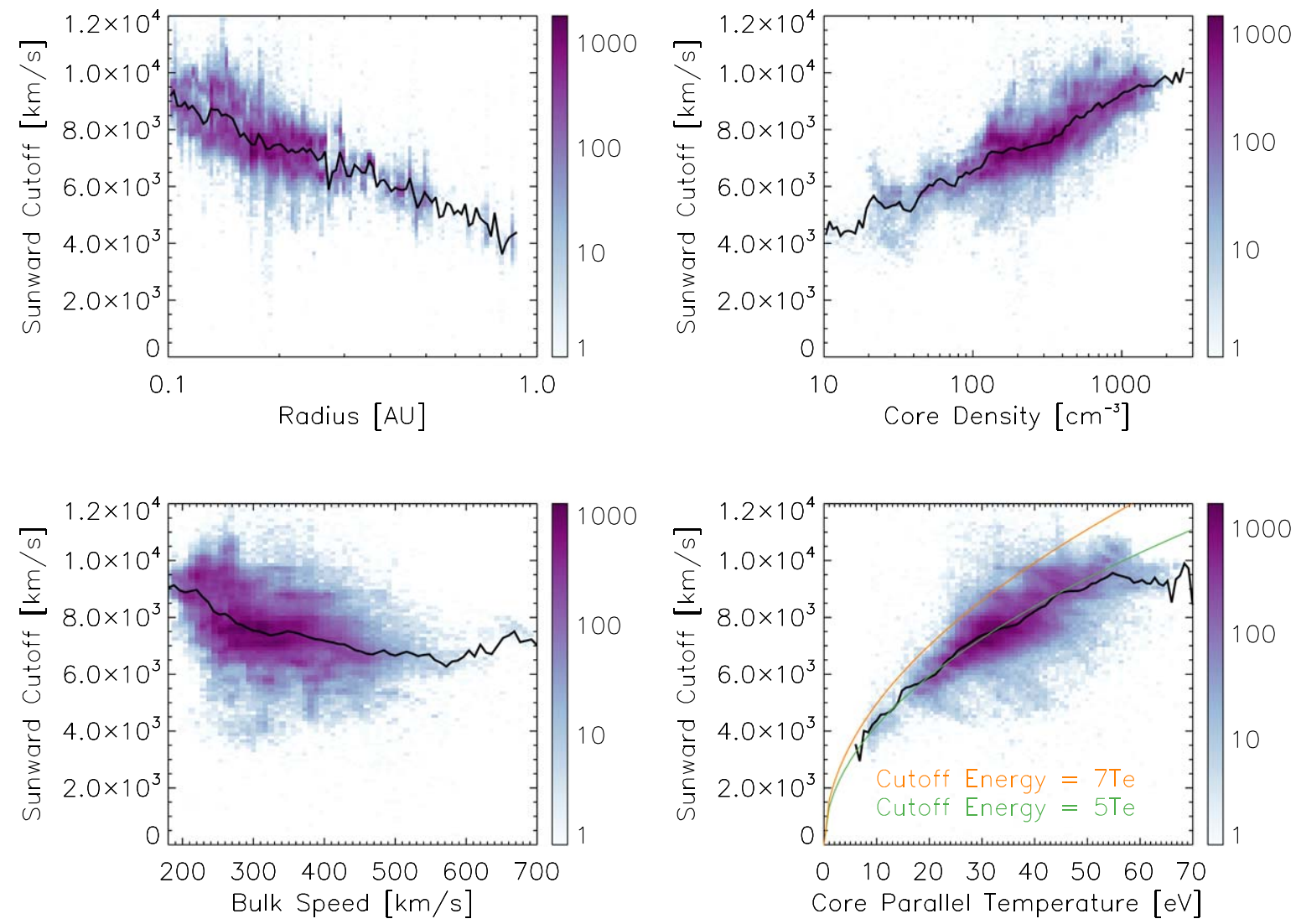

Figure 4. Histograms showing joint occurrence distributions of the local sunward electron cutoff velocity (where measured) with heliocentric radius, electron core density, solar wind proton bulk speed, and electron core parallel temperature. Black lines in each panel indicate the mean cutoff velocity. Orange and green lines in the bottom right panel show the expected cutoff velocity for two given ratios of the cutoff energy to the local core temperature.

wind observed near the Sun by PSP on average has a greater remaining interplanetary potential drop to pass through, and thus will undergo more subsequent acceleration after the observation point. Berčič et al. (2021a) explores the relationship between the inferred interplanetary electric field and the solar wind acceleration in more detail.

\section{The Occurrence of the Sunward Deficit}

We now further investigate the conditions under which we can observe a clear signature of the sunward deficit. Given the apparent anticorrelation between the deficit occurrence and the magnitude of the fractional halo density seen in Figure 3, we choose to explicitly compare the occurrence of the deficit in the sunward pitch angle bin to the occurrence of fractional halo densities $>1 \%$. We show the distributions of observations that satisfy these two conditions, in Figure 5. As noted in the time series observations of Figure 3, we most often observe the sunward deficit close to the Sun, in plasma with low electron $\beta$ and collisional age, and at times with more anisotropic electron core distributions. In contrast, we observe significant halo densities for the opposite set of conditions.

Most observations of the sunward deficit come from heliocentric distances $<0.25 \mathrm{au}$, within the closest approach of Helios, explaining why PSP has provided the first clear reports of this signature. Clear signatures of the sunward deficit occur in $\sim 60 \%-80 \%$ of the observations within $\sim 0.2 \mathrm{au}$, but the fractional occurrence frequency falls to $\simeq 10 \%$ by $\sim 0.3$ au. At the same time, the occurrence of halo densities above our chosen $1 \%$ threshold rises from $\lesssim 20 \%$ to $\sim 60 \%$. Given that our halo fit procedure does not successfully converge for every VDF, the upper figure represents a lower bound. Even in the crossover range of radii, the simultaneous occurrence of the deficit and significant halo densities remains $\lesssim 15 \%$, lending credence to the hypothesis that the formation of the halo may obscure the presence of the deficit. Though the physical mechanism that leads to this rapid transition with radius remains unclear, it does not result from a change in the SPANE field of view, which remains constant with distance. Furthermore, the small field of view blockage of the PSP heat shield should not affect the result, as the sunward suprathermal deficit extends over a broad range of angles observed in the anti-sunward look directions of the sensors.

We find similar occurrence trends as a function of $\beta$, collisional age, and core anisotropy (as one would expect given the significant correlations between all of these parameters). For $\beta \lesssim 0.4, \mathrm{Ae} \lesssim 40$, and core anistropy $\lesssim 0.8, \sim 90 \%$ of the observed VDFs from within 0.5 au contain clear signatures of the sunward deficit. For $\beta \gtrsim 5, A e \gtrsim 100$, and core anistropy $\gtrsim 1.0, \lesssim 10 \%$ of the observed VDFs contain clear signatures of the sunward deficit.

These observed trends naturally motivate the question of whether a single process could simultaneously produce the halo and obscure the sunward deficit. Oblique whistler instabilities provide one natural candidate, as they can rapidly scatter the strahl over a broad angular range to form a halo-like distribution (Cattell \& Vo 2021). A multi-stage process whereby oblique whistlers scatter the strahl to form the halo, and then quasi-parallel whistlers redistribute the halo into a 

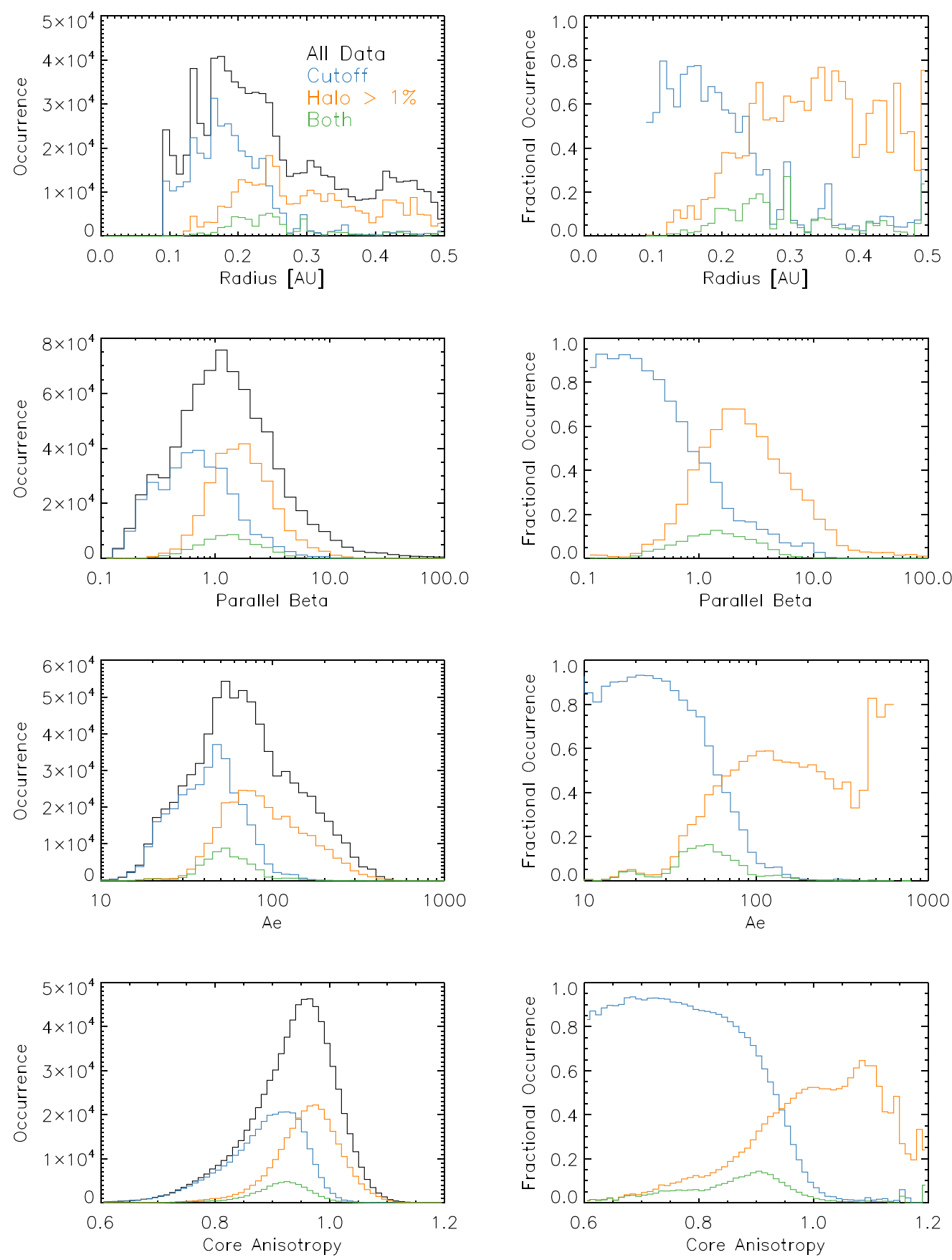

Figure 5. Absolute (left column) and normalized (right column) occurrence distributions for all electron VDFs analyzed from heliocentric distances $<0.5$ au (black), VDFs with an observable sunward deficit (blue), VDFs for which the halo comprises $>1 \%$ of the electron density (orange), and VDFs for which both of these conditions are satisfied (green), as a function of heliocentric radius, electron $\beta_{\|}$, collisional age, and core anisotropy.

more isotropic state (Micera et al. 2020), also provides a plausible hypothesis to explain these observations. Alternatively, an instability related to the sunward deficit itself could operate, as suggested by Berčič et al. (2021b). Malaspina et al. (2021) also discussed a loss cone instability driven by sunward electrons as a candidate to generate narrowband plasma waves observed by PSP near the Sun.

To further investigate the role of instabilities in erasing the signature of the deficit, we look at the occurrence distribution of the deficit in several spaces potentially relevant to candidate instabilities, as shown in Figure 6. These distributions do not obviously implicate a single candidate instability, but do have several interesting characteristics that may provide constraints on what instability or instabilities play important roles.

We first consider the possible role of core anisotropy-driven instabilities. Intriguingly, the transition between VDFs that mostly contain the deficit (dark purple colors) and those that do not (light gray colors) appears to follow a curve that lies nearly parallel to the $\beta$-dependent whistler anisotropy instability threshold (Gary \& Wang 1996) (for a growth rate of $0.01 \Omega_{e}$ ). The signature of the deficit disappears at core anisotropies well below the relevant threshold, so the correspondence appears imperfect. The relevant curve would move downward for a lower growth rate; however, we have already chosen a 

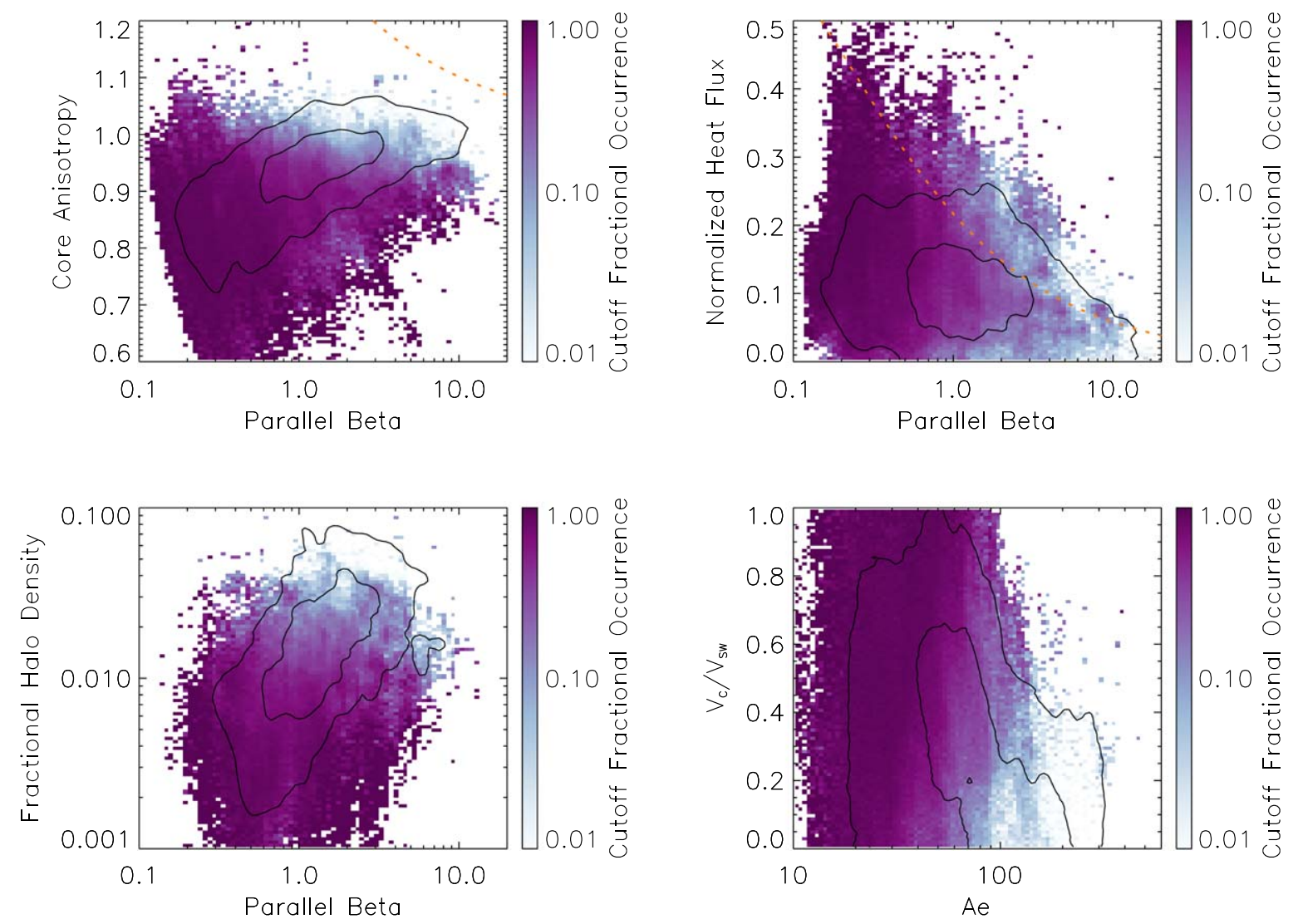

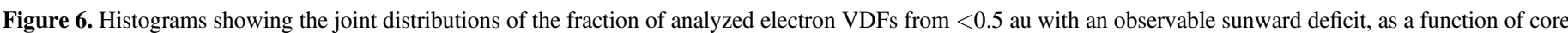

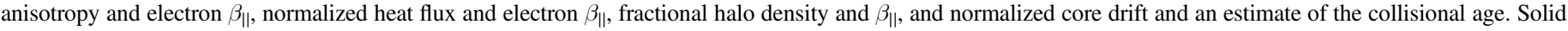

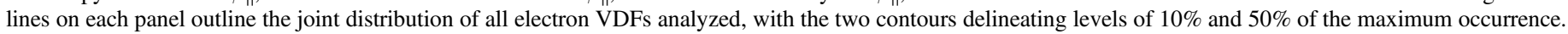
Dashed orange lines on the top two panels show thresholds for selected candidate instabilities.

conservatively small growth rate. On the other hand, we note that the presence of a deficit in the sunward direction may effectively increase the perpendicular anisotropy, as compared with a purely bi-Maxwellian core, and thereby lower the threshold for the whistler anisotropy instability. Along similar lines, Berčič et al. (2021b) have proposed a quasi-parallel whistler instability driven by the velocity gradient associated with the sunward deficit.

We next address the possible role of heat flux-driven instabilities. Surprisingly, though the frequency of observation of the deficit clearly correlates with $\beta$, we find no apparent organization by heat flux. We compare to the $\beta$-dependent oblique heat flux instability proposed by Vasko et al. (2019) (for $\Delta_{s} / v_{c}=1.5$ ), and find no evidence for any organization of the observations by this threshold or indeed any other $\beta$ dependent heat flux threshold. This seemingly argues against both the quasi-parallel whistler heat flux instability and oblique whistler/magnetosonic instabilities as primary mechanisms to erase the deficit.

We also investigate the occurrence of the deficit in terms of fractional halo density and $\beta$. In accordance with the 1D distributions shown in Figure 5, we find that the deficit occurrence frequency decreases and the fractional halo density increases with increasing $\beta$. In this case, little new information is revealed by the 2D distribution shown in Figure 6.

Finally, we consider the possible effect of instabilities related to the electron core drift. The magnitude of the sunward core drift, as compared with the solar wind bulk flow velocity, decreases with increasing collisional age, possibly due to instabilities driven by the relative drift between the protons and electrons (Gary et al. 1975). The processes that act to reduce this relative drift may also help obscure the deficit, given that our observations indicate the deficit has a slightly lower occurrence frequency for smaller relative drifts, at a given collisional age.

\section{The Width of the Sunward Cutoff}

Many of the same processes that could work to obscure the deficit should also act to broaden the cutoff from its initial form (presumably a step function) to a more smoothly varying feature in the VDF. In this final section, we therefore investigate the width of the cutoff, using the parameter $w$ derived from our hyperbolic tangent fits. We show the results as a function of electron $\beta$ and collisional age in Figure 7 . We find a significant degree of variability in the results, which may result from the discrete SPAN-E energy binning and/or the simplicity of the functional form we chose to represent the shape of the cutoff. Nonetheless, we do find clear trends in the width of the cutoff with both $\beta$ and collisional age, providing support for the hypothesis that the cutoff relaxes from an initial step function to a more gradual transition. Coulomb collisions provide a potential mechanism for this relaxation process (Boldyrev et al. 2020).

We find very similar trends both before and after normalizing the cutoff width by the value of the cutoff velocity. This provides confidence that the observed trends do not result solely from the instrumental increase in both energy resolution 

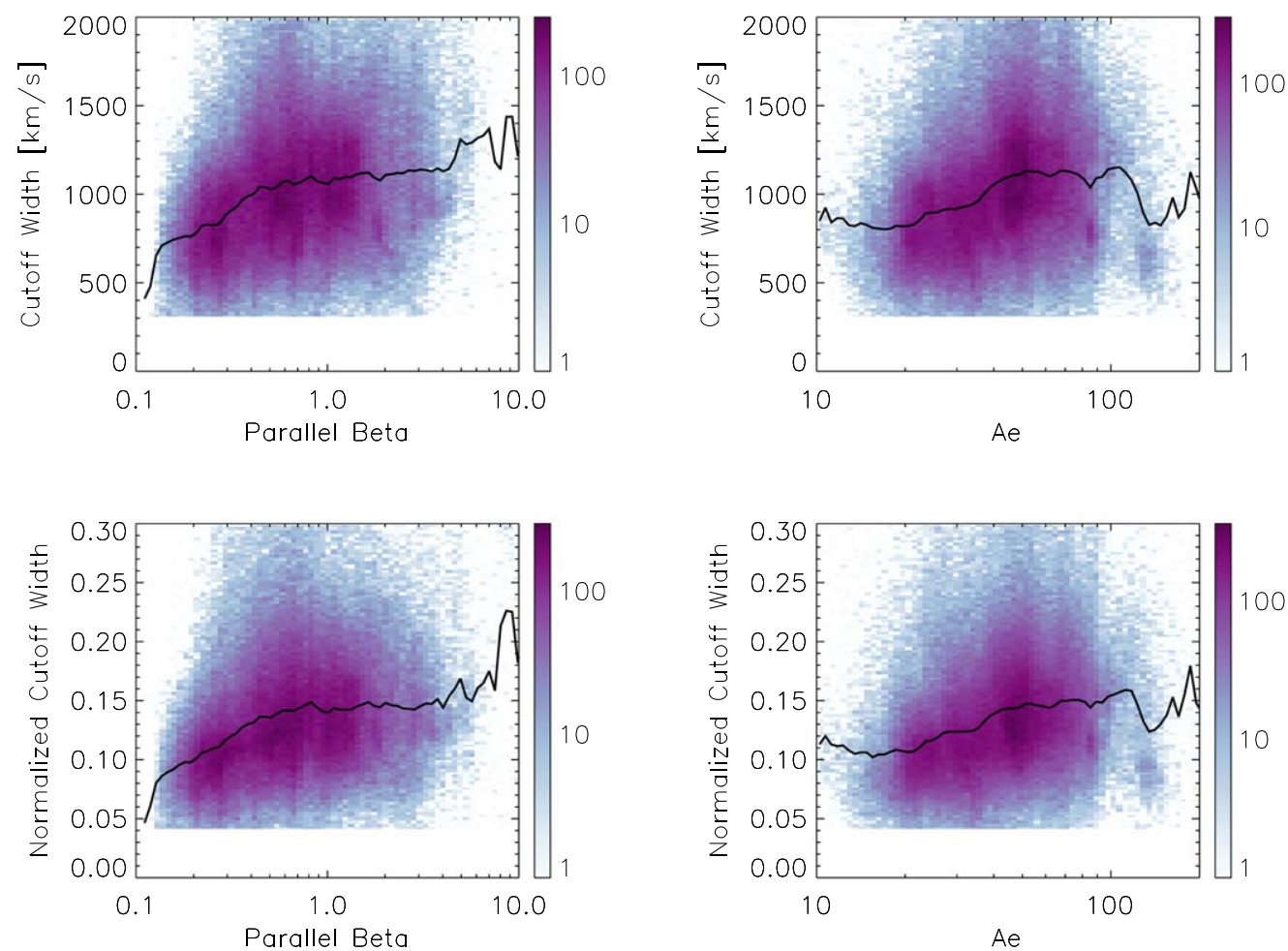

Figure 7. Histograms showing the joint occurrence distribution of the velocity width of the sunward electron cutoff (where measured, for VDFs from $<0.5$ au) with electron $\beta_{\|}$(left column) and an estimate of the collisional age (right column). The top panels show the cutoff width in velocity units, while the bottom panels show the width normalized by the cutoff velocity. We remove a small population of very narrow and non-physical cutoff widths resulting from misfits. Black lines in each panel indicate the mean values.

and energy bin size with energy. An instrumental resolution effect would lead to larger widths for larger cutoff velocities, which would generally act to flatten any physical trend in the normalized width. Such an instrumental artifact would also lead to larger average widths at low $\beta$ and collisional age, as these occur at smaller heliocentric distances and therefore correlate with larger cutoff velocities. We see just the opposite, indicating that the observed trends are robust.

\section{Conclusions and Implications}

As PSP travels closer to the Sun, and deeper into its electric potential, the electron VDFs we observe reveal new information about the heliosphere and the solar wind, much of which is not accessible at larger heliocentric distances. Some features of the electron VDF, such as the strahl and the heat flux it carries (Berčič et al. 2020; Halekas et al. 2021), and the core and the anticorrelation of its temperature with bulk speed (Maksimovic et al. 2020; Halekas et al. 2020), provide information about the initial conditions in the corona. Other characteristics of the electron VDF, such as those that we discuss in this work, inform us about the processes that impact the evolution of the solar wind in the inner heliosphere. The PSP electron measurements for the first time reveal a clear signature of the Sun's electric potential, in the form of a deficit in the sunward suprathermal electron population. We can use the location of the lower boundary of this deficit in phase space (the cutoff velocity) to recover the structure of the electric potential drop between the corona and the outer heliosphere, with clear implications for the acceleration of the solar wind. This potential, $\sim 50-300 \mathrm{~V}$ at the location of PSP, though significant in comparison to solar wind particle energies, corresponds to an electric field on the order of $\mathrm{nV} / \mathrm{m}$, not measurable by even state of the art in situ electric field instruments. Berčič et al. (2021a) looks in more detail at how much of the solar wind acceleration this potential can provide.

The sunward deficit provides relevant information not only through its magnitude, but also through its structure and its presence or absence. The fact that the sunward deficit often extends to locally mirroring pitch angles or even into the antisunward angles indicates that the electron core population undergoes significant nonadiabatic pitch angle diffusion, likely facilitated by the combined trapping effect of the magnetic mirror force and the interplanetary electric field. Intriguingly, the persistence and angular range of the deficit together suggest that pitch angle diffusion must occur more rapidly than energy diffusion, in contrast to predictions based only on Coulomb collisions (Boldyrev et al. 2020), and thus potentially indicating a role for plasma instabilities in shaping the core.

Though energy diffusion apparently operates less rapidly than pitch angle diffusion, it does occur, as evidenced by the increasing width of the cutoff with both $\beta$ and collisional age. By $\sim 0.2-0.3 \mathrm{au}$, obvious signatures of the sunward deficit largely disappear. This eventual obscuration of the deficit could result from the same energy diffusion and/or from the operation of processes that produce the suprathermal halo. The clear anticorrelation of significant fractional halo densities with the presence of the deficit suggests that the formation of the halo may play an important role in erasing the sunward deficit. A plausible scenario might involve oblique instabilities that rapidly scatter the strahl over a broad angular range (Cattell \& Vo 2021), producing the halo and obscuring the deficit simultaneously. However, the fact that the probability of observing the deficit correlates not with the electron heat flux, but with core temperature anisotropy, complicates the story. 
This observation may instead point toward a role for quasiparallel whistler instabilities that occur as a secondary consequence of the initial scattering of the strahl by oblique instabilities (Micera et al. 2020) and/or as a direct result of the presence of the deficit itself (Berčič et al. 2021b). The processes that regulate the relative drift between the electrons and protons may also play a role, though the electron core drift also ultimately ties to the heat flux, through current balance.

In any case, the presence of the sunward deficit near the Sun implies that we may need to reconsider our typical models for the electron VDF. Rather than modeling the electron core as a simple convecting bi-Maxwellian, we should consider a truncated function as a better representation of the VDF. This alteration of the nominally assumed structure of the VDF may also change the details of the instability calculations often performed, leading to changes in the relative importance of solar wind electron instabilities and their thresholds, or even to the existence of instabilities that do not typically operate at greater heliocentric distances.

We acknowledge the SWEAP contract NNN06AA01C for support.

\section{ORCID iDs}

J. S. Halekas (i) https://orcid.org/0000-0001-5258-6128

L. Berčič (i) https://orcid.org/0000-0002-6075-1813

P. Whittlesey (1) https://orcid.org/0000-0002-7287-5098

D. E. Larson (1) https://orcid.org/0000-0001-5030-6030

R. Livi (i) https://orcid.org/0000-0002-0396-0547

M. Berthomier (1) https://orcid.org/0000-0001-6235-5382

J. C. Kasper (1) https://orcid.org/0000-0002-7077-930X

A. W. Case (1) https://orcid.org/0000-0002-3520-4041

M. L. Stevens (i) https://orcid.org/0000-0002-7728-0085

S. D. Bale (i) https://orcid.org/0000-0002-1989-3596

R. J. MacDowall (i) https://orcid.org/0000-0003-3112-4201

M. P. Pulupa (1) https://orcid.org/0000-0002-1573-7457

\section{References}

Bale, S. D., Balikhin, M. A., Horbury, T. S., et al. 2005, SSRv, 118, 161 Bale, S. D., Goetz, K., Harvey, P. R., et al. 2016, SSRv, 204, 49 Berčič, L., Landi, S., \& Maksimović, M. 2021, JGRA, 126, e28864 Berčič, L., Larson, D., Whittlesey, P., et al. 2020, ApJ, 892, 88 Berčič, L., Maksimovic, M., Halekas, J. S., et al. 2021a, ApJ, submitted Berčič, L., Maksimović, M., Landi, S., \& Matteini, L. 2019, MNRAS, 486, 3404

Berčič, L., Verscharen, D., Owen, C. J., et al. 2021b, A\&A, in press Boldyrev, S., Forest, C., \& Egedal, J. 2020, PNAS, 117, 9232 Boldyrev, S., \& Horaites, K. 2019, MNRAS, 489, 3412

Case, A. W., Kasper, J. C., Stevens, M. L., et al. 2020, ApJS, 246, 43 Cattell, C., Breneman, A., Dombeck, J., et al. 2021, ApJL, 911, L29

Cattell, C., \& Vo, T. 2021, ApJL, 914, L33
Feldman, W. C., Asbridge, J. R., Bame, S. J., Montgomery, M. D., \& Gary, S. P. 1975, JGR, 80, 4181

Fox, N. J., Velli, M. C., Bale, S. D., et al. 2016, SSRv, 204, 7

Gary, S. P. 1978, JPlPh, 20, 47

Gary, S. P., Feldman, W. C., Forslund, D. W., \& Montgomery, M. D. 1975, JGR, 80, 4197

Gary, S. P., Scime, E. E., Phillips, J. L., \& Feldman, W. C. 1994, JGR, 99, 23391

Gary, S. P., Skoug, R. M., \& Daughton, W. 1999, PhPl, 6, 2607

Gary, S. P., \& Wang, J. 1996, JGR, 101, 10749

Graham, G. A., Rae, I. J., Owen, C. J., et al. 2017, JGRA, 122, 3858

Gringauz, K. I., Bezrokikh, V. V., Ozerov, V. D., \& Rybchinskii, R. E. 1960, $\mathrm{SPhD}, 5,361$

Halekas, J. S., Whittlesey, P., Larson, D. E., et al. 2020, ApJS, 246, 22

Halekas, J. S., Whittlesey, P. L., Larson, D. E., et al. 2021, A\&A, 650, A15

Hammond, C. M., Feldman, W. C., McComas, D. J., Phillips, J. L., \& Forsyth, R. J. 1996, A\&A, 316, 350

Horaites, K., Astfalk, P., Boldyrev, S., \& Jenko, F. 2018, MNRAS, 480, 1499 Horaites, K., Boldyrev, S., \& Medvedev, M. V. 2019, MNRAS, 484, 2474

Innocenti, M. E., Boella, E., Tenerani, A., \& Velli, M. 2020, ApJL, 898, L41 Jagarlamudi, V. K., Wit, T. D. d., Froment, C., et al. 2021, A\&A, 650, A9 Jockers, K. 1970, A\&A, 6, 219

Kasper, J. C., Abiad, R., Austin, G., et al. 2016, SSRv, 204, 131

Landi, S., Matteini, L., \& Pantellini, F. 2012, ApJ, 760, 143

Lemaire, J., \& Scherer, M. 1971, JGR, 76, 7479

Lemaire, J., \& Scherer, M. 1973, RvGeo, 11, 427

López, R. A., Lazar, M., Shaaban, S. M., Poedts, S., \& Moya, P. S. 2020, ApJ, 900, L25

López, R. A., Shaaban, S. M., Lazar, M., et al. 2019, ApJ, 882, L8

Maksimovic, M., Bale, S. D., Berčič, L., et al. 2020, ApJS, 246, 62

Maksimovic, M., Pierrard, V., \& Lemaire, J. F. 1997, A\&A, 324, 725

Maksimovic, M., Zouganelis, I., Chaufray, J.-Y., et al. 2005, JGRA, 110 A09104

Malaspina, D. M., Iii, L. B. W., Ergun, R. E., et al. 2021, A\&A, 650, A97

Micera, A., Zhukov, A. N., López, R. A., et al. 2020, ApJ, 903, L23

Moncuquet, M., Meyer-Vernet, N., Issautier, K., et al. 2020, ApJS, 246, 44

Neugebauer, M., \& Snyder, C. W. 1962, Sci, 138, 1095

Pannekoek, A. 1922, BAN, 1, 107

Parker, E. 1965, SSRv, 4, 666

Parker, E. N. 1958, ApJ, 128, 664

Parker, E. N. 2010, in AIP Proc. 1216, Twelfth Int. Solar Wind Conf. (Melville, NY: AIP), 3

Pierrard, V., \& Lemaire, J. 1996, JGR, 101, 7923

Pilipp, W. G., Miggenrieder, H., Montgomery, M. D., et al. 1987, JGRA, 92, 1075

Roberg-Clark, G. T., Drake, J. F., Swisdak, M., \& Reynolds, C. S. 2018, ApJ, 867,154

Rosenbauer, H., Schwenn, R., Marsch, E., et al. 1977, JGZG, 42, 561

Saeed, S., Sarfraz, M., Yoon, P. H., Lazar, M., \& Qureshi, M. N. S. 2017, MNRAS, 465, 1672

Salem, C., Hubert, D., Lacombe, C., et al. 2003, ApJ, 585, 1147

Scudder, J. D. 2019, ApJ, 885, 138

Scudder, J. D., \& Olbert, S. 1979a, JGR, 84, 2755

Scudder, J. D., \& Olbert, S. 1979b, JGR, 84, 6603

Shaaban, S. M., Lazar, M., \& Poedts, S. 2018, MNRAS, 480, 310

Vasko, I. Y., Krasnoselskikh, V., Tong, Y., et al. 2019, ApJ, 871, L29

Verscharen, D., Chandran, B. D. G., Jeong, S.-Y., et al. 2019, ApJ, 886, 136

Štverák, S., Maksimovic, M., Trávníček, P. M., et al. 2009, JGRA, 114, A05104

Whittlesey, P. L., Larson, D. E., Kasper, J. C., et al. 2020, ApJS, 246, 74

Zouganelis, I., Maksimovic, M., Meyer-Vernet, N., Lamy, H., \& Issautier, K. 2004, ApJ, 606, 542 